\title{
Transformation theory for phase-space representations of quantum mechanics
}

\author{
Joshua Wilkie \\ Department of Chemistry, Simon Fraser University, Burnaby, British Columbia, Canada V5A 1S6 \\ Paul Brumer \\ Chemical Physics Theory Group, Department of Chemistry, University of Toronto, Toronto, Ontario, Canada M5S 1A1
}

(Received 26 October 1999; published 4 May 2000)

\begin{abstract}
Dirac bras and kets are explicitly defined for phase-space representations of quantum mechanical observables, and simple transformation rules for changing representations are constructed. Derivations in the WignerWeyl representation of an expression for the kernel of the quantum Liouville operator, and of a relation between its stationary and nonstationary eigenstates, demonstrate the power of the resultant algebra.

PACS number(s): 03.65.-w
\end{abstract}

\section{INTRODUCTION}

Representation and transformation theory are central to formal quantum mechanics [1]. Phase-space representations (e.g., the Wigner-Weyl representation, the Husimi representation, etc.) are of considerable theoretical interest because they constitute a framework within which the formal differences between quantum and classical mechanics are minimized. For example, they provide an important framework within which the transition from quantum to classical physics can be understood [2,3]. However, formal manipulations in a phase-space representation are more difficult than analogous calculations employing coordinate and momentum representations in the traditional Hilbert space of wave functions due to the clumsiness of the associated algebra. For example, changing phase-space representations of an operator generally requires that one reconstruct the operator from its phase-space kernel, and then construct the kernel for the new representation. By contrast, calculations in the Hilbert space of wave functions are facilitated through the use of Dirac notation for states and their representations, and by simple rules governing a change of representation. The introduction of Dirac notation for the operators and superoperators [4] of the Liouville picture therefore greatly simplified formal algebraic manipulations, and recently the Dirac formalism has been extended to include certain phase-space representations, including the Wigner-Weyl representation [5]. However, the explicit construction of the dual space and of the rules for transforming between phase-space representations, arguably the most computationally useful aspect of Dirac notation, have not been developed.

In Sec. II of this paper we provide a simple set of rules for defining the appropriate bras and kets in these phase-space representations. We then show that the rules governing a change of representation emerge naturally from these definitions. In Sec. III we display the power of this approach by comparing expressions for the spectral decomposition of the quantum Liouville operator with and without the use of Dirac notation. We also utilize this transformation theory to derive an important identity in the Wigner-Weyl representation, and to show that a particularly simple expression for the kernel of the quantum Liouville operator follows from this identity.

\section{DIRAC FORMALISM FOR PHASE-SPACE REPRESENTATIONS}

\section{A. Bras and kets}

Consider a system with $s$ degrees of freedom. In conformity with the conventions of Dirac notation we choose the phase-space representation bras ( $\mathbf{x} \mid$ to denote linear functionals and kets $\mid \mathbf{x})$ to denote vectors. Here $\mathbf{x}=(\mathbf{p}, \mathbf{q})$, where $\mathbf{p}$ are the $s$ momenta and $\mathbf{q}$ are the $s$ coordinates. In the Liouville picture, linear functionals and vectors take the forms $\operatorname{Tr}\{\hat{C} \cdot\}$ and $\hat{D}$, respectively, where $\hat{C}$ and $\hat{D}$ are operators in the usual Schrödinger picture. Consequently, the bras and kets of a phase-space representation must take the forms

$$
\begin{gathered}
\left(\mathbf{x}_{\beta} \mid=\operatorname{Tr}\left\{\hat{B}_{\beta}^{\dagger}\left(\mathbf{x}_{\beta}\right) \cdot\right\},\right. \\
\left.\mid \mathbf{x}_{\beta}\right)=\hat{K}_{\beta}\left(\mathbf{x}_{\beta}\right) .
\end{gathered}
$$

Here Greek subscripts are used to denote the particular phase-space representation of interest, e.g., $\beta=W$ will denote the Wigner-Weyl representation, $\beta=H$ the Husimi representation, and $\beta=K$ the Kirkwood representation. Other representations may be treated in a similar manner to these, which are chosen as prime examples in this paper.

The operators $\hat{B}_{\beta}\left(\mathbf{x}_{\beta}\right)$ and $\hat{K}_{\beta}\left(\mathbf{x}_{\beta}\right)$, which generate the particular phase-space representation, depend parametrically on the phase-space variables $\mathbf{x}_{\beta}$ and are chosen so that $\left(\mathbf{x}_{\beta} \mid\right.$ and $\mid \mathbf{x}_{\beta}$ ) have the following properties: (a) dimensions of $\left[\right.$ action $^{-s / 2}$, (b) orthogonality, i.e.,

$$
\left(\mathbf{x}_{\beta} \mid \mathbf{x}_{\beta}^{0}\right)=\operatorname{Tr}\left\{\hat{B}_{\beta}^{\dagger}\left(\mathbf{x}_{\beta}\right) \hat{K}_{\beta}\left(\mathbf{x}_{\boldsymbol{\beta}}^{0}\right)\right\}=\delta\left(\mathbf{x}_{\beta}-\mathbf{x}_{\beta}^{0}\right),
$$

and (c) completeness [6]

$$
\left.\int d \mathbf{x}_{\beta} \mid \mathbf{x}_{\beta}\right)\left(\mathbf{x}_{\beta} \mid=1\right.
$$

The resulting bras $\left(\mathbf{x}_{\beta} \mid\right.$ generate representations $\left(\mathbf{x}_{\beta} \mid \rho\right)$ and $\left(\mathbf{x}_{\beta} \mid A\right)$ of the Hilbert space of physical states $\left.\mid \rho\right)$ $=h^{-s / 2} \hat{\rho}$ (where $\hat{\rho}$ is the density operator) and the associated space of operators (including observables) $\mid A)=h^{s / 2} \hat{A}$. The kets $\left.\mid \mathbf{x}_{\beta}\right)$ generate the $\beta$ phase-space representations $\left(\rho \mid \mathbf{x}_{\beta}\right)$ and $\left(A \mid \mathbf{x}_{\beta}\right)$ of the dual space of physical states $(\rho \mid$ $=h^{-s / 2} \operatorname{Tr}\left\{\hat{\boldsymbol{\rho}}^{\dagger} \cdot\right\}$ and the associated set of operators $(A \mid$ 
$=h^{s / 2} \operatorname{Tr}\left\{\hat{A}^{\dagger} \cdot\right\}$. The normalization of the states $\left.\left.\mid \rho\right), \mid A\right),(\rho \mid$, and $\left(A \mid\right.$ is chosen so that $\left(\mathbf{x}_{\beta} \mid \rho\right)$ and $\left(\rho \mid \mathbf{x}_{\beta}\right)$ have dimensions of [action] $]^{-s}$ and so that $\left(\mathbf{x}_{\beta} \mid A\right)$ and $\left(A \mid \mathbf{x}_{\beta}\right)$ have the same dimensions as $\hat{A}$. The representations $\left(\mathbf{x}_{\beta} \mid \rho\right),\left(\mathbf{x}_{\beta} \mid A\right)$ and $\left(\rho \mid \mathbf{x}_{\beta}\right),\left(A \mid \mathbf{x}_{\beta}\right)$ generally constitute independent representations, both of which can be used to compute expectations of observables. That is, the expectation value of $A$ in the state $\rho$ is given by

$$
\begin{aligned}
(A \mid \rho) & =\int d \mathbf{x}_{\beta}\left(A \mid \mathbf{x}_{\beta}\right)\left(\mathbf{x}_{\boldsymbol{\beta}} \mid \rho\right)=\operatorname{Tr}\left\{\hat{A}^{\dagger} \hat{\rho}\right\}=\left[\operatorname{Tr}\left\{\hat{\rho}^{\dagger} \hat{A}\right\}\right]^{*} \\
& =\left(\int d \mathbf{x}_{\beta}\left(\rho \mid \mathbf{x}_{\beta}\right)\left(\mathbf{x}_{\beta} \mid A\right)\right)^{*}=(\rho \mid A)^{*} .
\end{aligned}
$$

The generating operators $\hat{B}_{\beta}\left(\mathbf{x}_{\beta}\right)$ and $\hat{K}_{\beta}\left(\mathbf{x}_{\beta}\right)$ are discussed at length in Ref. [7]. For the Wigner $[5,7,8]$ representation $\hat{B}_{W}\left(\mathbf{x}_{W}\right)=\hat{K}_{W}\left(\mathbf{x}_{W}\right)=h^{-s / 2} \hat{\Delta}_{W}\left(\mathbf{x}_{W}\right)$, where the Hermitian operator $\hat{\Delta}_{W}\left(\mathbf{x}_{W}\right)$ takes the form

$$
\hat{\Delta}_{W}\left(\mathbf{x}_{W}\right)=h^{-s} \int d \mathbf{u} d \mathbf{v} e^{i\left[\mathbf{v} \cdot\left(\mathbf{p}_{W}-\hat{\mathbf{p}}\right)+\mathbf{u} \cdot\left(\mathbf{q}_{W}-\hat{\mathbf{q}}\right)\right] / \hbar} .
$$

The generating operators for the antinormal and normal $\mathrm{Hu}-$ simi [9] representations are, respectively, $\hat{B}_{H}\left(\mathbf{x}_{H}\right)$ $=h^{-s / 2} P_{\alpha} \hat{\Delta}_{H}\left(\mathbf{x}_{H}, \alpha\right)$ and $\hat{K}_{H}\left(\mathbf{x}_{H}\right)=h^{-s / 2} \hat{\Delta}_{H}\left(\mathbf{x}_{H}, \alpha\right)$. Here the Hermitian operator $\hat{\Delta}_{H}\left(\mathbf{x}_{H}, \alpha\right)$ is given by

$$
\begin{aligned}
\widehat{\Delta}_{H}\left(\mathbf{x}_{H}, \alpha\right)= & h^{-s} \int d \mathbf{u} d \mathbf{v} e^{i\left[\mathbf{v} \cdot\left(\mathbf{p}_{H}-\hat{\mathbf{p}}\right)+\mathbf{u} \cdot\left(\mathbf{q}_{H}-\hat{\mathbf{q}}\right)\right] / \hbar} \\
& \times e^{-\left(\alpha \mathbf{v}^{2}+\mathbf{u}^{2} / \alpha\right) / 4 \hbar}
\end{aligned}
$$

and the operator $P_{\alpha} \hat{\Delta}_{H}^{\dagger}\left(\mathbf{x}_{H}, \alpha\right)$ is defined as

$$
\left(\mathbf{x}_{H} \mid \rho\right)=P_{\alpha}\left(\rho \mid \mathbf{x}_{H}\right)=P_{\alpha} R\left(\mathbf{x}_{H}, \alpha\right)=R\left(\mathbf{x}_{H},-\alpha\right),
$$

where $R\left(\mathbf{x}_{H}, \alpha\right) \equiv\left(\rho \mid \mathbf{x}_{H}\right)$. The Kirkwood [7] representations are generated by the operators $\hat{B}_{K}\left(\mathbf{x}_{K}\right)=\hat{K}_{K}\left(\mathbf{x}_{K}\right)$ $=h^{-s / 2} \hat{\Delta}_{K}\left(\mathbf{x}_{K}\right)$ where

$$
\hat{\Delta}_{K}\left(\mathbf{x}_{K}\right)=h^{-s} \delta\left(\mathbf{p}_{K}-\hat{\mathbf{p}}\right) \delta\left(\mathbf{q}_{K}-\hat{\mathbf{q}}\right) .
$$

\section{B. Transformation rules and matrix elements}

As a result of defining the bras and kets in this manner, $a$ change of phase-space representation follows the same rules as does a change of representation for a wave function. For instance, suppose that we initially have $\hat{\rho}$ in the representation generated by $\hat{B}_{\beta}\left(\mathbf{x}_{\beta}\right)$, i.e., $\left(\mathbf{x}_{\beta} \mid \rho\right)=h^{-s / 2} \operatorname{Tr}\left\{\hat{B}_{\beta}\left(\mathbf{x}_{\beta}\right) \hat{\rho}\right\}$, and we wish to change to a representation generated by $\hat{B}_{\gamma}\left(\mathbf{x}_{\gamma}\right)$. Then,

$$
\left(\mathbf{x}_{\gamma} \mid \rho\right)=\int d \mathbf{x}_{\beta}\left(\mathbf{x}_{\gamma} \mid \mathbf{x}_{\beta}\right)\left(\mathbf{x}_{\beta} \mid \rho\right),
$$

where $\left(\mathbf{x}_{\gamma} \mid \mathbf{x}_{\beta}\right)$ is the transformation matrix element between the two representations (see Table I) given by $\operatorname{Tr}\left\{\hat{B}_{\gamma}^{\dagger}\left(\mathbf{x}_{\gamma}\right) \hat{K}_{\beta}\left(\mathbf{x}_{\beta}\right)\right\}$. Alternatively, starting with $\hat{\rho}$ in the representation generated by $\hat{K}_{\beta}\left(\mathbf{x}_{\beta}\right)$, i.e., $\left(\rho \mid \mathbf{x}_{\beta}\right)$ $=h^{s / 2} \operatorname{Tr}\left\{\hat{\rho} \hat{K}_{\beta}\left(\mathbf{x}_{\beta}\right)\right\}$, the change to a representation generated by $\hat{K}_{\gamma}\left(\mathbf{x}_{\gamma}\right)$ can be performed by

$$
\left(\rho \mid \mathbf{x}_{\gamma}\right)=\int d \mathbf{x}_{\beta}\left(\mathbf{x}_{\beta} \mid \mathbf{x}_{\gamma}\right)\left(\rho \mid \mathbf{x}_{\beta}\right),
$$

where $\left(\mathbf{x}_{\beta} \mid \mathbf{x}_{\gamma}\right)=\operatorname{Tr}\left\{\hat{B}_{\beta}^{\dagger}\left(\mathbf{x}_{\beta}\right) \hat{K}_{\gamma}\left(\mathbf{x}_{\gamma}\right)\right\}$. This representation theory can also be extended to include the $\mathbf{q}, \mathbf{q}^{\prime}$ and $\mathbf{p}, \mathbf{p}^{\prime}$ representations [that is, $\left(\mathbf{q}, \mathbf{q}^{\prime} \mid \rho\right)=h^{-s / 2}\left\langle\mathbf{q}|\hat{\rho}| \mathbf{q}^{\prime}\right\rangle$ and $\left.\left(\mathbf{p}, \mathbf{p}^{\prime} \mid \rho\right)=h^{-s / 2}\left\langle\mathbf{p}|\hat{\rho}| \mathbf{p}^{\prime}\right\rangle\right]$ of the density matrix [11] by defining operators

$$
\begin{gathered}
\hat{B}_{\mathbf{q}, \mathbf{q}^{\prime}}=\hat{K}_{\mathbf{q}, \mathbf{q}^{\prime}}=|\mathbf{q}\rangle\left\langle\mathbf{q}^{\prime}\right|, \\
\hat{B}_{\mathbf{p}, \mathbf{p}^{\prime}}=\hat{K}_{\mathbf{p}, \mathbf{p}^{\prime}}=|\mathbf{p}\rangle\left\langle\mathbf{p}^{\prime}\right| .
\end{gathered}
$$

Then, one may change from a $\mathbf{q}, \mathbf{q}^{\prime}$ or $\mathbf{p}, \mathbf{p}^{\prime}$ representation to a phase-space representation or vice versa by application of Eqs. (10) and (11). Other representations can easily be constructed. The transformation matrix elements $\left(\mathbf{x}_{\beta} \mid \mathbf{x}_{\gamma}\right)$ for the various representations discussed here are listed in Table I.

Particular care must be taken for the Husimi representation. Note first that for all but the Husimi representation $\left(\mathbf{x}_{\beta} \mid \mathbf{x}_{\gamma}\right)=\left(\mathbf{x}_{\gamma} \mid \mathbf{x}_{\beta}\right)^{*}$. Second, to use the $\left(\mathbf{p}_{H}, \mathbf{q}_{H} \mid \mathbf{x}_{\beta}\right)$ matrix elements to transform $\left(\mathbf{x}_{\beta} \mid \rho\right)$ or $\left(\mathbf{x}_{\beta} \mid A\right)$ to the Husimi representation requires that the integral over $\mathbf{x}_{\beta}$ be performed before the $P_{\alpha}$ operation. Similarly, to use $\left(\mathbf{p}_{H}, \mathbf{q}_{H} \mid \mathbf{x}_{\beta}\right)$ to transform $\left(\rho \mid \mathbf{p}_{H}, \mathbf{q}_{H}\right)$ or $\left(A \mid \mathbf{p}_{H}, \mathbf{q}_{H}\right)$ from the Husimi representation requires that the operation $P_{\alpha}$ be performed before the integral over $\mathbf{p}_{H}, \mathbf{q}_{H}$.

The approach introduced here is sufficiently general that it may be applied, with minor modifications, to phase-space representations in classical mechanics [10].

\section{SAMPLE PHASE-SPACE MANIPULATIONS}

\section{A. Spectral decomposition of the von Neumann equation}

To illustrate the advantages of our approach we compare the Dirac and density matrix expressions for the spectral decomposition of the quantum Liouville operator.

Let $|n\rangle$ be the complete, orthonormal set of eigenstates of a Hamiltonian $\hat{H}$, i.e., $\hat{H}|n\rangle=E_{n}|n\rangle,\langle n \mid m\rangle=\delta_{n, m}$, and $\Sigma_{n}|n\rangle\langle n|=1$. From these states we may construct basis states $\mid n, m)=|n\rangle\langle m|$ which play an analogous role in the Hilbert space associated with the eigensolutions of the timeindependent von Neumann equation

$$
\left.L \mid n, m)=\lambda_{n, m} \mid n, m\right),
$$

where $L=(1 / \hbar)[\hat{H}, \cdot]$ is the quantum Liouville operator, and $\lambda_{n, m}=\left(E_{n}-E_{m}\right) / \hbar$. Since the states $|n\rangle$ are complete and orthonormal, the states $\mid n, m)$ are also complete,

$$
\left.\sum_{n, m} \mid n, m\right)(n, m \mid=1
$$

and orthonormal,

$$
(n, m \mid k, l)=\delta_{n, m} \delta_{k, l} .
$$


TABLE I. Phase-space basis inner products $\left(\mathbf{x}_{\beta} \mid \mathbf{x}_{\gamma}\right)$. For example, the first element in the table is for $\beta=$ Husimi, $\gamma=$ Wigner.

\begin{tabular}{|c|c|}
\hline$\left(\mathbf{x}_{\beta} \mid \mathbf{x}_{\gamma}\right)$ & Wigner \\
\hline Husimi & $2{ }^{s} h^{-s} P_{\alpha} e^{-\left(\mathbf{p}_{W}-\mathbf{p}_{H}\right)^{2} / \alpha \hbar} e^{-\alpha\left(\mathbf{q}_{W}-\mathbf{q}_{H}\right)^{2} / \hbar}$ \\
\hline Kirkwood & $2^{s} h^{-s} e^{-2 i\left(\mathbf{q}_{W}-\mathbf{q}_{K}\right) \cdot\left(\mathbf{p}_{W}-\mathbf{p}_{K}\right) / \hbar}$ \\
\hline Coordinate & $h^{-s / 2} \delta\left(\mathbf{q}_{W}-\left(\mathbf{q}+\mathbf{q}^{\prime}\right) / 2\right) e^{-i\left(\mathbf{q}^{\prime}-\mathbf{q}\right) \cdot \mathbf{p}_{W} / \hbar}$ \\
\hline \multirow[t]{2}{*}{ Momentum } & $h^{-s / 2} \delta\left(\mathbf{p}_{W}-\left(\mathbf{p}+\mathbf{p}^{\prime}\right) / 2\right) e^{i\left(\mathbf{p}^{\prime}-\mathbf{p}\right) \cdot \mathbf{q}_{W} / \hbar}$ \\
\hline & Husimi \\
\hline Wigner & $2^{s} h^{-s} e^{-\left(\mathbf{p}_{W}-\mathbf{p}_{H}\right)^{2} / \alpha \hbar} e^{-\alpha\left(\mathbf{q}_{W}-\mathbf{q}_{H}\right)^{2} / \hbar}$ \\
\hline Kirkwood & $\begin{array}{c}2^{s / 2} h^{-s} e^{-i\left(\mathbf{q}_{H}-\mathbf{q}_{K}\right) \cdot\left(\mathbf{p}_{H}-\mathbf{p}_{K}\right) / \hbar} e^{-\left(\mathbf{p}_{H}-\mathbf{p}_{K}\right)^{2} / 2 \alpha \hbar} \\
\times e^{-\alpha\left(\mathbf{q}_{H}-\mathbf{q}_{K}\right)^{2} / 2 \hbar}\end{array}$ \\
\hline Coordinate & $\begin{array}{c}(2 \alpha)^{s / 2} h^{-s} e^{-i\left(\mathbf{q}^{\prime}-\mathbf{q}\right) \cdot \mathbf{p}_{H} / \hbar} e^{-\alpha\left(\mathbf{q}^{\prime}-\mathbf{q}\right)^{2} / 4 \hbar} \\
\times e^{-\alpha\left[\mathbf{q}_{H}-\left(\mathbf{q}^{\prime}+\mathbf{q}\right) / 2\right]^{2} / \hbar}\end{array}$ \\
\hline \multirow[t]{2}{*}{ Momentum } & $\begin{array}{c}(2 / \alpha)^{s / 2} h^{-s} e^{-i\left(\mathbf{p}^{-} \mathbf{p}^{\prime}\right) \cdot \mathbf{q}_{H} / \hbar} e^{-\left(\mathbf{p}^{\prime}-\mathbf{p}\right)^{2} / 4 \alpha \hbar} \\
\times e^{-\left[\mathbf{p}_{H}-\left(\mathbf{p}^{\prime}+\mathbf{p}\right) / 2\right]^{2} / \alpha \hbar}\end{array}$ \\
\hline & Kirkwood \\
\hline Wigner & $2^{s} h^{-s} e^{2 i\left(\mathbf{q}_{W}-\mathbf{q}_{K}\right) \cdot\left(\mathbf{p}_{W}-\mathbf{p}_{K}\right) / \hbar}$ \\
\hline Husimi & $\begin{array}{c}2^{s / 2} h^{-s} P_{\alpha} e^{i\left(\mathbf{q}_{H}-\mathbf{q}_{K}\right) \cdot\left(\mathbf{p}_{H}-\mathbf{p}_{K}\right) / \hbar} e^{-\left(\mathbf{p}_{H}-\mathbf{p}_{K}\right)^{2} / 2 \alpha \hbar} \\
\times e^{-\alpha\left(\mathbf{q}_{H}-\mathbf{q}_{K}\right)^{2} / 2 \hbar}\end{array}$ \\
\hline Coordinate & $h^{-s / 2} \delta\left(\mathbf{q}_{K}-\mathbf{q}\right) e^{-i\left(\mathbf{q}^{\prime}-\mathbf{q}\right) \cdot \mathbf{p}_{K} / \hbar}$ \\
\hline \multirow[t]{2}{*}{ Momentum } & $h^{-s / 2} \delta\left(\mathbf{p}_{K}-\mathbf{p}^{\prime}\right) e^{i\left(\mathbf{p}^{\prime}-\mathbf{p}\right) \cdot \mathbf{q}_{K} / \hbar}$ \\
\hline & Coordinate \\
\hline Wigner & $h^{-s / 2} \delta\left(\mathbf{q}_{W}-\left(\mathbf{q}+\mathbf{q}^{\prime}\right) / 2\right) e^{i\left(\mathbf{q}^{\prime}-\mathbf{q}\right) \cdot \mathbf{p}_{W} / \hbar}$ \\
\hline Husimi & $\begin{array}{c}(2 \alpha)^{s / 2} h^{-s} P_{\alpha} e^{i\left(\mathbf{q}^{\prime}-\mathbf{q}\right) \cdot \mathbf{p}_{H} / \hbar} e^{-\alpha\left(\mathbf{q}^{\prime}-\mathbf{q}\right)^{2} / 4 \hbar} \\
\times e^{-\alpha\left[\mathbf{q}_{H}-\left(\mathbf{q}^{\prime}+\mathbf{q}\right) / 2\right]^{2} / \hbar}\end{array}$ \\
\hline Kirkwood & $h^{-s / 2} \delta\left(\mathbf{q}_{K}-\mathbf{q}\right) e^{i\left(\mathbf{q}^{\prime}-\mathbf{q}\right) \cdot \mathbf{p}_{K} / \hbar}$ \\
\hline \multirow[t]{2}{*}{ Momentum } & $h^{-s} e^{-i\left(\mathbf{q} \cdot \mathbf{p}-\mathbf{q}^{\prime} \cdot \mathbf{p}^{\prime}\right) / \hbar}$ \\
\hline & Momentum \\
\hline Wigner & $h^{-s / 2} \delta\left(\mathbf{p}_{W}-\left(\mathbf{p}+\mathbf{p}^{\prime}\right) / 2\right) e^{-i\left(\mathbf{p}^{\prime}-\mathbf{p}\right) \cdot \mathbf{q}_{W} / \hbar}$ \\
\hline Husimi & $\begin{array}{c}(2 / \alpha)^{s / 2} h^{-s} P_{\alpha} e^{i\left(\mathbf{p}^{-}-\mathbf{p}^{\prime}\right) \cdot \mathbf{q}_{H} / \hbar} e^{-\left(\mathbf{p}^{\prime}-\mathbf{p}\right)^{2} / 4 \alpha \hbar} \\
\times e^{-\left[\mathbf{p}_{H}-\left(\mathbf{p}^{\prime}+\mathbf{p}\right) / 2\right]^{2} / \alpha \hbar}\end{array}$ \\
\hline Kirkwood & $h^{-s / 2} \delta\left(\mathbf{p}_{K}-\mathbf{p}^{\prime}\right) e^{-i\left(\mathbf{p}^{\prime}-\mathbf{p}\right) \cdot \mathbf{q}_{K} / \hbar}$ \\
\hline Coordinate & $h^{-s} e^{i\left(\mathbf{q} \cdot \mathbf{p}^{-} \mathbf{q}^{\prime} \cdot \mathbf{p}^{\prime}\right) / \hbar}$ \\
\hline
\end{tabular}

Thus, the operators $\mid n, m)(n, m \mid$ are spectral projection operators and the quantum Liouville operator can be decomposed in the form

$$
\left.L=\sum_{n, m} \lambda_{n, m} \mid n, m\right)(n, m \mid
$$

To satisfy orthonormality the linear functionals $(n, m \mid$ must take the form $\left(n, m \mid=\operatorname{Tr}\left\{(|n\rangle\langle m|)^{\dagger} \cdot\right\}\right.$. The density matrix analog of Eq. (17) therefore takes the ungainly form

$$
L=\sum_{n, m} \lambda_{n, m}|n\rangle\langle m| \operatorname{Tr}\{|m\rangle\langle n| \cdot\} .
$$

In a phase-space representation the kernel of the Liouville spectral decomposition takes the form

$$
\left(\mathbf{x}_{\beta}|L| \mathbf{x}_{\beta}^{0}\right)=\sum_{n, m} \lambda_{n, m}\left(\mathbf{x}_{\beta} \mid n, m\right)\left(n, m \mid \mathbf{x}_{\beta}^{0}\right)
$$

in Dirac notation, while the analogous expression in density matrix notation is

$$
\left(\mathbf{x}_{\beta}|L| \mathbf{x}_{\beta}^{0}\right)=\sum_{n, m} \lambda_{n, m}\left\langle m\left|\hat{B}^{\dagger}\left(\mathbf{x}_{\beta}\right)\right| n\right\rangle\left\langle n\left|\hat{K}\left(\mathbf{x}_{\beta}^{0}\right)\right| m\right\rangle .
$$

The Liouville spectral decomposition as expressed in Eqs. (18) and (20) is far from obvious and could not be easily obtained without the formalism introduced in Sec. II.

\section{B. Stationary and nonstationary Liouville eigenfunctions}

The relationship between the stationary $\mid n, n)(n, n \mid$ and nonstationary $\mid n, m)(n, m \mid$ eigenfunctions of the Liouville operator in the Wigner-Weyl representation plays a central role in the proof that classical mechanics emerges from quantum mechanics in the classical limit for chaotic systems [3]. In addition, as shown below, this expression leads to a useful formula for the kernel of the Liouville operator. Specifically, the phase-space relationship is

$$
\begin{aligned}
\left(\mathbf{x}_{W} \mid n, m\right)\left(n, m \mid \mathbf{x}_{W}^{0}\right) \\
=h^{-s} \int d \mathbf{y} e^{i\left(\mathbf{x}_{W}-\mathbf{x}_{W}^{0}\right) \cdot J \cdot \tilde{\mathbf{y}} / \hbar}\left(\mathbf{x}_{W}+\mathbf{y} / 2 \mid n, n\right) \\
\\
\quad \times\left(m, m \mid \mathbf{x}_{W}^{0}-\mathbf{y} / 2\right),
\end{aligned}
$$

where $\mathbf{x}_{W}=\left(\mathbf{p}_{W}, \mathbf{q}_{W}\right), \mathbf{x}_{W}^{0}=\left(\mathbf{p}_{W}^{0}, \mathbf{q}_{W}^{0}\right), \mathbf{y}=(\mathbf{u}, \mathbf{v})$, and

$$
J=\left(\begin{array}{cc}
0 & -I \\
I & 0
\end{array}\right)
$$

is the $2 s \times 2 s$ dimensional symplectic matrix. This is the Wigner-Weyl representation of the $\mathbf{q}, \mathbf{q}^{\prime}$ representation expression:

$$
\begin{aligned}
\left(\mathbf{q}, \mathbf{q}^{\prime} \mid n, m\right)\left(n, m \mid \mathbf{q}_{0}, \mathbf{q}_{0}^{\prime}\right) & =\left\langle\mathbf{q}^{\prime} \mid m\right\rangle\langle n \mid \mathbf{q}\rangle\left\langle\mathbf{q}_{0} \mid n\right\rangle\left\langle m \mid \mathbf{q}_{0}^{\prime}\right\rangle \\
& =\left(\mathbf{q}_{0}, \mathbf{q} \mid n, n\right)\left(m, m \mid \mathbf{q}^{\prime}, \mathbf{q}_{0}^{\prime}\right) .
\end{aligned}
$$

Formula (21) requires a great deal of effort to verify, and the task of deriving Eq. (21) from Eq. (22) without employing the representation theory discussed above is quite challenging. By contrast, Eq. (21) can easily be obtained from Eq. (22) using the closure relations and inner product formulas for the phase-space representations introduced above.

Specifically, consider $\left(\mathbf{x}_{W} \mid n, m\right)\left(n, m \mid \mathbf{x}_{W}^{0}\right)$. Inserting closure relations for $\left.\mid \mathbf{q}, \mathbf{q}^{\prime}\right)$ and $\left.\mid \mathbf{q}_{0}, \mathbf{q}_{0}^{\prime}\right)$ gives

$$
\begin{aligned}
\left(\mathbf{x}_{W} \mid n, m\right)\left(n, m \mid \mathbf{x}_{W}^{0}\right)= & \int d \mathbf{q} d \mathbf{q}^{\prime} d \mathbf{q}_{0} d \mathbf{q}_{0}^{\prime}\left(\mathbf{x}_{W} \mid \mathbf{q}, \mathbf{q}^{\prime}\right) \\
& \times\left(\mathbf{q}, \mathbf{q}^{\prime} \mid n, m\right)\left(n, m \mid \mathbf{q}_{0}, \mathbf{q}_{0}^{\prime}\right) \\
& \times\left(\mathbf{q}_{0}, \mathbf{q}_{0}^{\prime} \mid \mathbf{x}_{W}^{0}\right) .
\end{aligned}
$$

Inserting Eq. (22) we obtain 


$$
\begin{aligned}
\left(\mathbf{x}_{W} \mid n, m\right)\left(n, m \mid \mathbf{x}_{W}^{0}\right)= & \int d \mathbf{q} d \mathbf{q}^{\prime} d \mathbf{q}_{0} d \mathbf{q}_{0}^{\prime}\left(\mathbf{x}_{W} \mid \mathbf{q}, \mathbf{q}^{\prime}\right) \\
& \times\left(\mathbf{q}_{0}, \mathbf{q} \mid n, n\right)\left(m, m \mid \mathbf{q}^{\prime}, \mathbf{q}_{0}^{\prime}\right) \\
& \times\left(\mathbf{q}_{0}, \mathbf{q}_{0}^{\prime} \mid \mathbf{x}_{W}^{0}\right) .
\end{aligned}
$$

The goal now is to rearrange terms until closure relations for $\left.\mid \mathbf{q}_{0}, \mathbf{q}\right)$ and $\left.\mid \mathbf{q}^{\prime}, \mathbf{q}_{0}^{\prime}\right)$ can be removed.

Using Table I to obtain expressions for the matrix elements $\left(\mathbf{q}_{0}, \mathbf{q}_{0}^{\prime} \mid \mathbf{x}_{W}^{0}\right)$ and $\left(\mathbf{x}_{W} \mid \mathbf{q}, \mathbf{q}^{\prime}\right)$, and inserting the identity $1=\int d \mathbf{v} \delta\left(\mathbf{v}-\mathbf{q}_{0}+\mathbf{q}^{\prime}\right)$ gives, after some rearrangement, the relation

$$
\begin{aligned}
&\left(\mathbf{x}_{W} \mid n, m\right)\left(n, m \mid \mathbf{x}_{W}^{0}\right) \\
&=h^{-s} \int d \mathbf{q} d \mathbf{q}^{\prime} d \mathbf{q}_{0} d \mathbf{q}_{0}^{\prime} d \mathbf{v} \\
& \times \delta\left(\mathbf{v}-\mathbf{q}_{0}+\mathbf{q}^{\prime}\right) \cdot \delta\left(\mathbf{q}_{W}+\mathbf{v} / 2-\left(\mathbf{q}_{0}+\mathbf{q}\right) / 2\right) \\
& \times e^{i\left(\mathbf{q}_{0}-\mathbf{q}-\mathbf{v}\right) \cdot \mathbf{p}_{W} / \hbar}\left(\mathbf{q}_{0}, \mathbf{q} \mid n, n\right) \cdot\left(m, m \mid \mathbf{q}^{\prime}, \mathbf{q}_{0}^{\prime}\right) \\
& \times \delta\left(\mathbf{q}_{W}^{0}-\mathbf{v} / 2-\left(\mathbf{q}^{\prime}+\mathbf{q}_{0}^{\prime}\right) / 2\right) e^{-i\left(\mathbf{q}_{0}^{\prime}-\mathbf{q}^{\prime}-\mathbf{v}\right) \cdot \mathbf{p}_{W}^{0} / \hbar} .
\end{aligned}
$$

Rewriting $\quad \delta\left(\mathbf{v}-\mathbf{q}_{0}+\mathbf{q}^{\prime}\right)=h^{-s} \int d \mathbf{u} \exp \left[-i\left(\mathbf{v}-\mathbf{q}_{0}\right.\right.$ $\left.\left.+\mathbf{q}^{\prime}\right) \cdot \mathbf{u} / \hbar\right]$, adding the identities $1=\exp \left\{i\left[\mathbf{q}_{W}+\mathbf{v} / 2-\left(\mathbf{q}_{0}\right.\right.\right.$ $+\mathbf{q}) / 2] \cdot \mathbf{u} / \hbar\}$ and $1=\exp \left\{-i\left[\mathbf{q}_{W}^{0}-\mathbf{v} / 2-\left(\mathbf{q}^{\prime}+\mathbf{q}_{0}^{\prime}\right) / 2\right] \cdot \mathbf{u} / \hbar\right\}$ inside the integrand, and rearranging factors gives

$$
\begin{aligned}
\left(\mathbf{x}_{W} \mid n, m\right)\left(n, m \mid \mathbf{x}_{W}^{0}\right) & \\
= & h^{-s} \int d \mathbf{y} e^{i\left(\mathbf{x}_{W}-\mathbf{x}_{W}^{0}\right) \cdot J \cdot \tilde{\mathbf{y}} / \hbar} \int d \mathbf{q}_{0} d \mathbf{q}\left(\mathbf{x}_{W}+\mathbf{y} / 2 \mid \mathbf{q}_{0}, \mathbf{q}\right) \\
& \times\left(\mathbf{q}_{0}, \mathbf{q} \mid n, n\right) \cdot \int d \mathbf{q}^{\prime} d \mathbf{q}_{0}^{\prime}\left(m, m \mid \mathbf{q}^{\prime}, \mathbf{q}_{0}^{\prime}\right) \\
& \times\left(\mathbf{q}^{\prime}, \mathbf{q}_{0}^{\prime} \mid \mathbf{x}_{W}^{0}-\mathbf{y} / 2\right) .
\end{aligned}
$$

The desired expression [Eq. (21)] is obtained upon removal of the closure relations for $\left.\mid \mathbf{q}_{0}, \mathbf{q}\right)$ and $\mid \mathbf{q}^{\prime}, \mathbf{q}_{0}^{\prime}$ ).

Equation (21) also provides a means of obtaining a particularly interesting integral formula for the kernel of the quantum Liouville operator in the Wigner-Weyl representation. Substituting Eq. (21) into Eq. (19) gives

$$
\begin{aligned}
\left(\mathbf{x}_{W}|L| \mathbf{x}_{W}^{0}\right)= & h^{-s} \int d \mathbf{y} e^{i\left(\mathbf{x}_{W}-\mathbf{x}_{W}^{0}\right) \cdot J \cdot \tilde{\mathbf{y}} / \hbar} \cdot \sum_{n, m}\left(E_{n}-E_{m}\right) / \hbar \\
& \times\left(\mathbf{x}_{W}+\mathbf{y} / 2 \mid n, n\right)\left(m, m \mid \mathbf{x}_{W}^{0}-\mathbf{y} / 2\right) .
\end{aligned}
$$

The sums over $n$ and $m$ can be performed explicitly. $\Sigma_{m}\left(m, m \mid \mathbf{x}_{W}^{0}-\mathbf{y} / 2\right)=h^{-s / 2} ; \quad \Sigma_{n}\left(\mathbf{x}_{W}+\mathbf{y} / 2 \mid n, n\right)=h^{-s / 2}$; $\Sigma_{m}\left(m, m \mid \mathbf{x}_{W}^{0}-\mathbf{y} / 2\right)=h^{-s / 2} H\left(\mathbf{x}_{W}^{0}-\mathbf{y} / 2\right) ; \quad \Sigma_{n}\left(\mathbf{x}_{W}+\mathbf{y} / 2 \mid n, n\right)$ $=h^{-s / 2} H\left(\mathbf{x}_{W}+\mathbf{y} / 2\right)$, giving

$$
\begin{aligned}
\left(\mathbf{x}_{W}|L| \mathbf{x}_{W}^{0}\right)= & h^{-2 s} \hbar^{-1} \int d \mathbf{y} e^{i\left(\mathbf{x}_{W}-\mathbf{x}_{W}^{0}\right) \cdot J \cdot \tilde{\mathbf{y}} / \hbar}\left[H\left(\mathbf{x}_{W}+\mathbf{y} / 2\right)\right. \\
& \left.-H\left(\mathbf{x}_{W}^{0}-\mathbf{y} / 2\right)\right],
\end{aligned}
$$

which is a particularly simple integral formula for the kernel of the quantum Liouville operator. That is, using Eq. (28) one can readily verify that

$$
\int d \mathbf{x}_{W}^{0}\left(\mathbf{x}_{W}|L| \mathbf{x}_{W}^{0}\right)\left(\mathbf{x}_{W}^{0} \mid \rho\right)=L\left(\mathbf{x}_{W}\right)\left(\mathbf{x}_{W} \mid \rho\right)
$$

where $\quad L\left(\mathbf{x}_{W}\right)=(2 i / \hbar) H\left(\mathbf{x}_{W}\right) \sin \left[(\hbar / 2)\left(\partial \leftarrow / \partial \mathbf{x}_{W}\right) \cdot J \cdot(\widetilde{\partial \rightarrow}\right.$ $\left.\left.\partial \mathbf{x}_{W}\right)\right]$ is the Liouville operator in the Wigner-Weyl representation. In the correspondence limit $h \rightarrow 0$ Eq. (28) reduces to the kernel of the classical Liouville operator $\left(\mathbf{x}\left|L_{c}\right| \mathbf{x}^{0}\right)$ $=i[\partial H(\mathbf{x}) / \partial \mathbf{x}] \cdot J \cdot(\tilde{\partial} / \partial \mathbf{x}) \delta\left(\mathbf{x}-\mathbf{x}^{0}\right)$.

\section{CONCLUSIONS}

In summary, we have introduced a Dirac notation for the phase-space representations of the quantum density matrix, and shown how rules governing the change of phase-space representations follow from these definitions. The resulting transformation rules bear a close resemblance to the rules for a canonical transformation of the classical phase-space probability density. The general transformation theory introduced here is expected to be useful in a wide variety of areas where phase-space representations of quantum mechanics have proven useful.

\section{ACKNOWLEDGMENT}

This work was supported by the Natural Sciences and Engineering Research Council of Canada.
[1] P.A.M. Dirac, The Principles of Quantum Mechanics (Oxford University Press, Oxford, 1958).

[2] C. Jaffé and P. Brumer, J. Chem. Phys. 82, 2330 (1985); C. Jaffe et al., Phys. Rev. Lett. 54, 8 (1985).

[3] J. Wilkie and P. Brumer, Phys. Rev. A 55, 27 (1997); 55, 43 (1997)

[4] U. Fano, Rev. Mod. Phys. 29, 74 (1957).

[5] A. Royer, Phys. Rev. A 43, 44 (1991); S. Mukamel, Principles of Nonlinear Optical Spectroscopy (Oxford University Press, New York, 1995).

[6] For the particular case of the Husimi representation, the domain of validity of the closure relation is restricted due to the fact that, for trace class operators $\hat{O}$, the representation $(\mathbf{x} \mid O)$ may be nonintegrable. This difficulty can be surmounted by using only the normal-ordered representation $(\rho \mid \mathbf{x})$ for physical states $\hat{\rho}$ (which are in fact trace class), and the antinormalordered representation $(\mathbf{x} \mid A)$ for observables.

[7] N.L. Balazs and B.K. Jennings, Phys. Rep. 104, 347 (1984).

[8] S.R. De Groot and L.G. Suttorp, Foundations of Electrodynamics (North-Holland, Amsterdam, 1972).

[9] S.S. Mizrahi, Physica A 127, 241 (1984).

[10] H. Goldstein, Classical Mechanics (Addison-Wesley, New York, 1950).

[11] Note that $\mathbf{q}, \mathbf{p}$ representations are trivially related to the Kirkwood representation, i.e., $K_{K}\left(\mathbf{x}_{K}\right)=e^{i \mathbf{p}_{K} \cdot \mathbf{q}_{K} / \hbar}\left|\mathbf{q}_{K}\right\rangle\left\langle\mathbf{p}_{K}\right|$. 